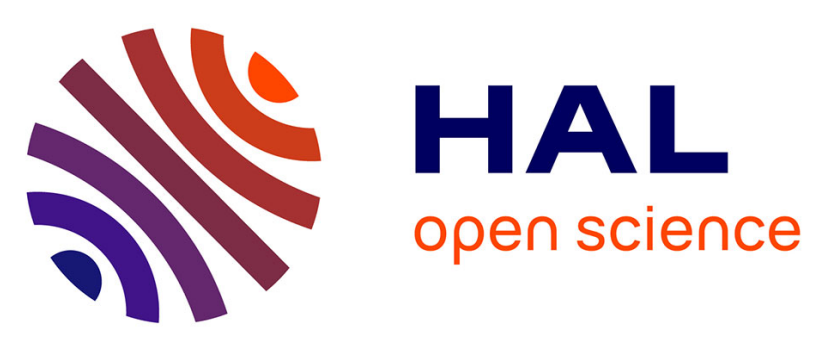

\title{
Inter-individual registration and dose mapping for voxe-lwise population analysis of rectal toxicity in prostate cancer radiotherapy.
}

Gaël Dréan, Oscar Acosta, Caroline Lafond, Antoine Simon, Renaud de Crevoisier, Pascal Haigron

\section{To cite this version:}

Gaël Dréan, Oscar Acosta, Caroline Lafond, Antoine Simon, Renaud de Crevoisier, et al.. Interindividual registration and dose mapping for voxe-lwise population analysis of rectal toxicity in prostate cancer radiotherapy.. Medical Physics, 2016, 43 (6), pp.2721. hal-01365309

\author{
HAL Id: hal-01365309 \\ https://hal.science/hal-01365309
}

Submitted on 11 Dec 2016

HAL is a multi-disciplinary open access archive for the deposit and dissemination of scientific research documents, whether they are published or not. The documents may come from teaching and research institutions in France or abroad, or from public or private research centers.
L'archive ouverte pluridisciplinaire HAL, est destinée au dépôt et à la diffusion de documents scientifiques de niveau recherche, publiés ou non, émanant des établissements d'enseignement et de recherche français ou étrangers, des laboratoires publics ou privés. 
Inter-individual registration and dose mapping for voxel-wise population analysis of rectal toxicity in prostate cancer radiotherapy Gaël Dréan ${ }^{1,2}$, Oscar Acosta ${ }^{1,2, *}$, Caroline Lafond ${ }^{1,2,3}$, Antoine Simon $^{1,2}$, Renaud de Crevoisier ${ }^{1,2,3}$ and Pascal Haigron ${ }^{1,2}$

\section{Abstract}

Purpose: Recent studies revealed a trend towards voxel-wise population analysis in order to understand the local dose/toxicity relationships in prostate cancer radiotherapy. Such approaches require, however, an accurate inter-individual mapping of the anatomies and 3D dose distributions towards a common coordinate system. This step is challenging due to the high inter-individual variability. In this paper, we propose a method designed for interindividual non-rigid registration of the rectum and dose mapping for population analysis.

Methods: The method is based on the computation of a normalized structural description of the rectum using a Laplacian-based model. This description takes advantage of the tubular structure of the rectum embedded in a non-rigid registration-based scheme. The performances of the method were evaluated on 30 individuals treated for prostate cancer in a leave-one-out cross validation.

Results: Performance was measured using classical metrics (Dice score and Hausdorff distance), along with new metrics devised to better assess dose mapping in relation with structural deformation (dose-organ overlap). Considering these scores, the proposed method outperforms intensity-based and distance maps-based registration methods.

Conclusion: the proposed method allows for accurately mapping inter-individual 3D dose distributions towards a single anatomical template, opening the way for further voxel-wise statistical analysis. 
32

Several studies have shown that increasing the dose to the prostate leads to improved local cancer control $(1,2)$. However, such dose increases are limited by treatment-related toxicity involving the organs at risk $(3,4)$. Understanding dose/toxicity relationship in prostate cancer radiotherapy proves to be crucial for improving treatment reliability from the planning stage onward. Normal tissue complication probability (NTCP) toxicity prediction models like the Lyman-Kutcher-Burman model $(5,6)$ have been developed in order to predict toxicity events using dosimetric data. The main observations considered are dose-volume histograms (DVHs), which provide an overall representation of dose distribution based on the dose delivered per percentage of organ volume. In particular, these models have rendered it possible to establish dose-volume constraints during treatment planning so as to limit the occurrence of unwanted events (7). Nevertheless, these models display limitations $(8,9)$, for instance those relating to the use of DVHs that result in a loss of anatomical spatial information like the organ shape and 3D dose distribution.

The strong correlation that may exist between different dose parameters of a DVH makes it difficult to extract the dose measurement that is the closest correlated with toxicity events. Furthermore, the models do not provide any understanding of local relationships between dose and effect at a suborgan level. Based on the hypothesis that certain regions of healthy organs may be more sensitive than others, new models aiming at spatially analyzing the relationship between dose and toxicity have been suggested. Some models have sought to geometrically describe spatial dose distribution on the rectal wall and correlate this description with the occurrence of rectal toxicity events (10-13). However, the parametric description of dose was approximative, and only the dose to the organ surface was taken into account.

More recently, approaches with in-depth analysis of 3D dose distribution using voxel-wise population analysis techniques have been proposed (14-16). These techniques aim to analyze the dose-effect relationship in each voxel across a population with the objective of identifying anatomical regions that may be responsible for the toxicity event occurrence. They necessitate several steps: (i) the mapping of a population of individuals in terms of their anatomy by non-rigid registration onto an anatomical template; (ii) propagation of dose distributions according to the anatomical transformation thus obtained; (iii) a local statistical analysis of dose-effect relationship. The performance of such an approach depends, however, on the accuracy of the anatomical mapping. This step may be performed with the computed tomography (CT) images used for computing the 3D dose distribution. 
Interindividual mapping of the pelvic region using computed tomography (CT) planning images has been the subject of very little research in the literature. It appears complex, particularly given the very high interindividual anatomical variability (organ volume, presence or absence of gas in the rectum, etc.) and low contrast of soft tissues $(17,18)$.

In the setting of radiotherapy, research into the anatomical registration of CT images has mainly concentrated on intraindividual images, i.e., per-treatment cone beam CT and planning CT for dose accumulation purposes (19-21). Algorithms have been based on intensity for the most part $(19,22-24)$. However, given the low contrast between soft tissues and due to the high anatomical variation observed even in the intrapatient setting, methods seeking to combine image intensity information with organ segmentation have been proposed $(21,25,26)$, using, for example, distinctive anatomical points such as calcifications, salient points, markers or distance map combined with organs meshes to guide image matching $(20,27-31)$.

In the interindividual setting, conventional registration methods based solely on the grayscale of CT images do not enable sufficiently accurate mapping $(17,18)$. Adding other information like anatomical features to guide the image matching process may render it possible to improve mapping accuracy in population analysis applications. Such methods are yet to be explored in settings where anatomical differences are greater and where greater deformations are being considered. See supplementary material at [URL will be inserted by AIP] in Figure 7 (electronic version), which depicts delineations and planning dose distributions for two different individals. Employing structural descriptor maps that exploit the rectum's pseudocylindrical nature may allow the rectums of different individuals to be described in a comparable manner, rather than using tissue intensity as displayed by CT images. Note should also be taken of the steep dose gradient within the rectum, which introduces an additional difficulty as a small matching error may result in a very large dosimetric error.

The main contribution of our paper is to propose an anatomical mapping method for the rectum that employs non-rigid image registration based on a 3D structural model of the rectum constructed using delineations that were made on treatment planning CT scans during routine clinical practice. This model makes use of the pseudocylindrical nature of the 
organ at risk of interest. It is founded on the solution of Laplace's equation (32), as estimated between the centerline and surface of the rectum. What is more, mapping accuracy was assessed from not only an anatomical view point but also a dosimetric one, using two new metrics that assess rectal centerline dispersion during the registration process and organ overlap relative to dose distribution.

We then present the construction method for a structural description based on the Laplacian operator in section II.1 followed by the method of registration and dose propagation into the common reference system in sections II.2 and II.3. We deal with the assessment metrics in section II.4. Results are presented and discussed in sections III and IV.

\section{Methods}

The principle of the method proposed for registering the rectum $R_{i}$ and propagating the dose $d_{i}$ of the individual $i$ to the rectum of the anatomical template $R_{A T}$ is described in Figure 1. Step 1 involved calculating a 3D structural model $\Phi_{i}$ and $\Phi_{A T}$ of $R_{i}$ and $R_{A T}$, respectively, using the centerline and Laplacian field $u$. Next (step 2 ), following a rigid registration that optimized the sum of squared differences (SSD) and served as an initialization, a Demons nonrigid transformation aligned the individual's rectal model $\Phi_{i}$ with the anatomical template rectal model $\Phi_{A T}$. Once the nonrigid transformation $\vec{T}=\overrightarrow{D F}$ 。 $T_{\text {rigid }}$ had been obtained, where $\overrightarrow{D F}$ represented the deformation field resulting from nonrigid registration, two propagation processes were implemented (step 3); one concerned the rectum $R_{i}$, and the other concerned dose distribution $d_{i}$. Thus in the end the dose $d_{i}$ of the individual was mapped onto the anatomical template reference system. 


\section{Individual $i$}

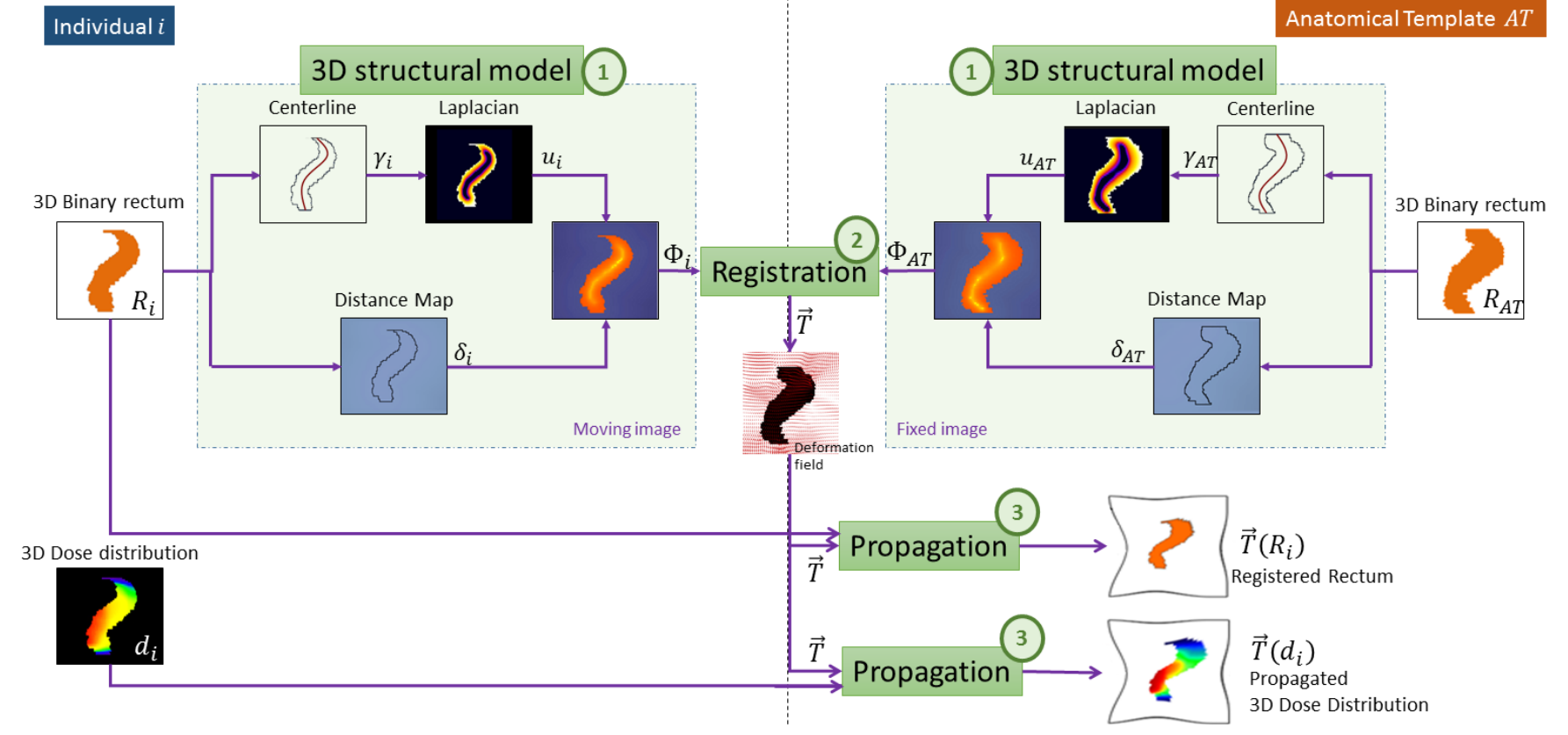

Figure 1: Proposed method for mapping the rectum and 3D dose distribution of an individual to the common anatomical template (AT).

We will first describe the structural descriptors for the rectum. We will then move on to the interindividual mapping process before finishing with an assessment of the proposed method's performance.

\section{III.1 Construction of a 3D structural model of the rectum}

To structurally describe a number of rectums of a population, it is essential that the same portion of the rectum be delineated according to a single clinical protocol. Here, organs were delineated by a specialist according to the same radiotherapy planning protocol, namely limiting the rectum to $2 \mathrm{~cm}$ above and below the prostate and seminal vesicles. Like a cylinder, which can be entirely described by its radius and generatrix, the rectum, a pseudocylindrical tubular structure, may be described structurally based on the organ's surface and centerline.

\section{a. Centerline $\gamma_{i}$}

Numerous methods exist in the literature for calculating the centerline of a structure, including skeletonization $(33,34)$, minimal-path $(35,36)$ and flux-driven techniques $(37)$. We chose the skeletonization method proposed by (34), which preserves the structure's topology and involves homotopically thinning a structure by iteratively deleting points in ascending order of distance map value as calculated within the object. This method was 
used to calculate the centerlines $\gamma$ of rectums following image smoothing using a Gaussian filter, which blurs irregularities arising from interslice manual segmentation.

\section{b. Structural descriptor map: Laplacian field $\boldsymbol{u}$ and distance map $\delta$}

The structural descriptors planned for registering the rectum were: (i) Laplacian field as estimated between the rectal boundary $F_{E x t}$ and centerline $\gamma$ in order to describe the inside of the organ $(32,38)$ and (ii) the map of Euclidean distances to the rectal boundary $\delta$ in order to describe the outside of the organ (39). The method is detailed below, where it is abbreviated to DMLA for Distance Map/Laplacian.

Solving Laplace's equation in a discrete domain has led to several possibilities for characterizing various structures in medical imaging. For example, the determination of organ thickness proposed by Jones $(32,38)$ was used to accurately calculate thickness $(40,41)$.

In this paper, the scalar field $u$ solution of Laplace's equation as calculated within the rectum between the centerline and the surface $(42,43)$ enables the rectum's cylindrical structure to be described in a similar fashion. Thus, registration can be driven by direct point-to-point correspondences within each individual's rectum. demarcated by an external boundary $F_{E x t}$, here the rectal surface, and an internal boundary, here the rectal centerline $\gamma$. Its mathematical form is:

$$
\Delta u(x, y, z)=\frac{\partial^{2} u}{\partial x^{2}}+\frac{\partial^{2} u}{\partial y^{2}}+\frac{\partial^{2} u}{\partial z^{2}}=0
$$

with the Dirichlet boundary conditions: $u(x, y, z)=u_{E x t}$ if $(x, y, z) \in F_{E x t}$ and $u(x, y, z)=u_{\gamma}$ if $(x, y, z) \in \gamma$, where $\left\{u_{E x t}, u_{\gamma}\right\} \in \mathbb{R}$. isopotential surfaces that form a smooth transition between $F_{E x t}$ and $\gamma$ and respect the domain's geometry. 

$(40,44)$. To avoid resampling anisotropic images, Laplace's equation was iteratively

$$
\begin{gathered}
u_{i t+1}(x, y, z)=\frac{1}{2\left(s_{y}^{2} s_{z}^{2}+s_{x}^{2} s_{z}^{2}+s_{x}^{2} s_{y}^{2}\right)}\left(s_{y}^{2} s_{z}^{2}\left[u_{i t}\left(x+s_{x}, y, z\right)+u_{i t}\left(x-s_{x}, y, z\right)\right]\right. \\
+s_{x}^{2} s_{z}^{2}\left[u_{i t}\left(x, y+s_{y}, z\right)+u_{i t}\left(x, y-s_{y}, z\right)\right] \\
\left.+s_{x}^{2} s_{y}^{2}\left[u_{i t}\left(x, y, z+s_{z}\right)+u_{i t}\left(x, y, z-s_{z}\right)\right]\right)
\end{gathered}
$$

with spacing $s_{x}, s_{y}$ and $s_{z}$ respectively; $u_{i t}$ the solution, i.e. the potential, calculated on the $i t^{\text {th }}$ iteration.

In our implementation, the Dirichlet conditions on $F_{E x t}$ and $\gamma$ were set at 0 on the centerline and 10000 on the rectal boundary to limit errors arising from calculation approximations, while intensities on $R \backslash\left\{F_{E x t}, \gamma\right\}$ were set at 5000, i.e., the mean, as proposed in previous implementations (43). See supplementary material at [URL will be inserted by AIP], Figure 8 $(a, b)$ (electronic version) which depicts the estimation of Laplace field in two rectums is illustrated in

Dose-effect relationships will not be analyzed outside the rectum, as the issue being dealt with here is local dose relationships within the organ as well as rectal side-effects. However, a comparable description of the outside of each individual's rectum is needed in order to guide transformation during registration. The outside of the rectum was here described using an Euclidean distance map $\delta$ to the rectal surface (39).

Thus, taking an anatomical template $A T$ and an individual $i$ to be mapped, descriptor maps $\left(\Phi_{k}, k \in\{i, M A R\}\right)$ were constructed according to the equation:

$$
\Phi_{k}(v)=\left\{\begin{array}{l}
u_{k}(v), \text { if } v \in R_{k} \\
\delta_{k}(v) \text { otherwise }
\end{array} \quad, \forall k \in\{i, A T\}\right.
$$

with $u_{k}$ and $\delta_{k}$ the solution field of Laplace's equation and the distance map as calculated for the individual $k \in\{i, A T\}$. 
To calculate the transformation $D F$ pulling the individual's rectal structural model onto the anatomical template's rectal structural model, we used the accelerated Demons iterative algorithm of (24). The Demons algorithm offers the advantage of being easily configurable and has shown good performance in the intrapatient registration of $\mathrm{CT} /$ relatively wellcontrasted CT images (19,22-24). The algorithm is based on a calculation of forces from optical flow. Registration comprises an energy function minimization problem between the rectal structural model of the anatomical template $\Phi_{A T}$ and that of the individual $\Phi_{i}$. This energy function is a trade-off between deformation regularity as effected by applying a Gaussian filter and image similarity, usually defined by the SSD:

$$
\mathrm{DF}=\arg \min _{T}\left\{S S D\left(D F\left(\Phi_{i}\right), \Phi_{A T}\right)\right\}
$$

Transformation is calculated using forces based on the gradient of the images:

$$
\begin{gathered}
F_{e}(x)=\frac{\left(D F\left(\Phi_{i}\right)(x)-\Phi_{A T}(x)\right) \vec{\nabla} \Phi_{A T}}{\left|\vec{\nabla} \Phi_{A T}\right|^{2}+\left(D F\left(\Phi_{i}\right)(x)-\Phi_{A T}(x)\right)^{2}} \\
F_{i}(x)=-\frac{\left(D F\left(\Phi_{i}\right)(x)-\Phi_{A T}(x)\right) \vec{\nabla} T\left(\Phi_{i}\right)}{\left|\vec{\nabla} D F\left(\Phi_{i}\right)\right|^{2}+\left(D F\left(\Phi_{i}\right)(x)-\Phi_{A T}(x)\right)^{2}}
\end{gathered}
$$

where $F_{e}$ represents an external force as calculated in a voxel $x$ and where $F_{i}$ represents an internal force that deforms the fixed image into the floating image by bringing into play the floating image's gradient. Given that the maps of the descriptors $\Phi_{i}$ and $\Phi_{A T}$ were constructed via the Laplace field, the values of $D F\left(\Phi_{i}\right)(x)$ and $\Phi_{A T}(x)$ were comparable, thus permitting cost function convergence based on the SSD.

In our implementation, and in the interest of speed, the algorithm was repeated four times in a coarse-to-fine multiresolution scheme so as to initially estimate the major deformations found in an interindividual setting. The images were then undersampled by a factor of two between each level following Gaussian kernel smoothing, the standard deviation of which was set at the size of a voxel. Figure 2 depicts an example of the registration as a deformation field and resulting jacobian determinant map. This map represents how each voxel deformed in term of its volumetric change, greater than one in case of volume expansion and less than one for volume contraction. 


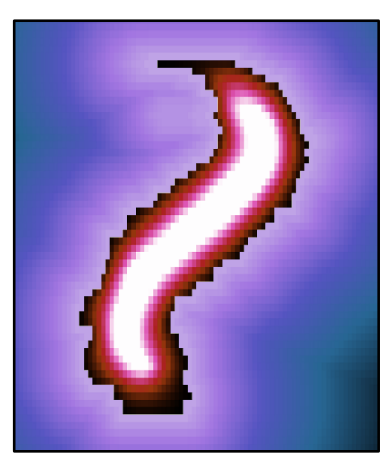

a) Individual I $\Phi_{i}$

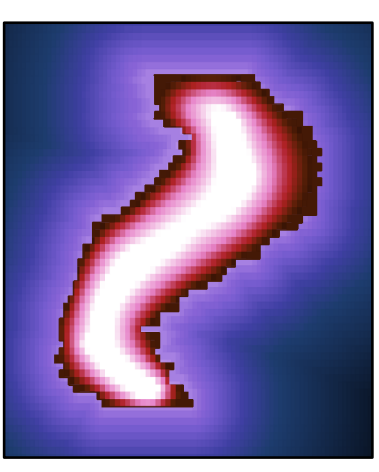

b) Anatomical template $\Phi_{A T}$

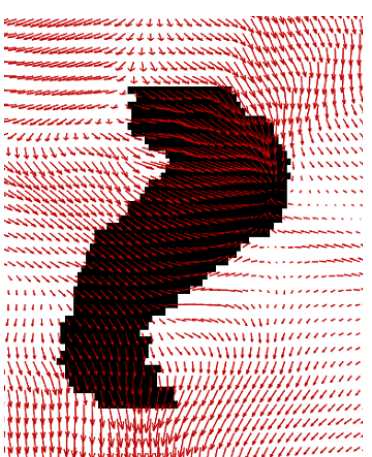

c) Deformation field (DF)

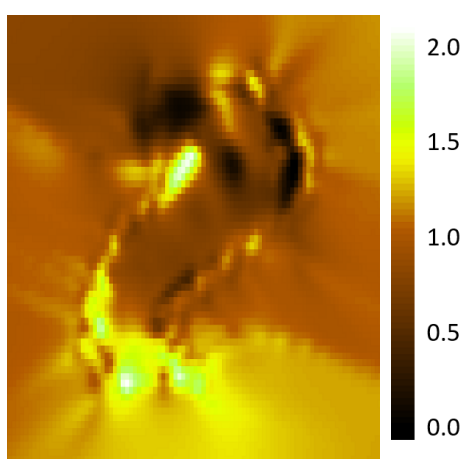

d) Jacobian Determinant map.

Figure 2. a) Individual structural model being non-rigidly registered to an anatomical template (b). c) obtained deformation field and d) Jacobian Determinant map illustrating regions of volmetric changes (expansion of contraction).

\section{III.3 Propagation of dose}

Once the deformation field $D F$ was calculated by non-rigid registration, binary images of the rectums $R_{i}$ derived from manual delineations were propagated into the anatomical template space using the calculated transformation $T=D F \circ T_{\text {Rigid }}$ and nearest-neighbor interpolation in order to preserve the image's binary nature. Similarly, dose distribution was propagated using trilinear interpolation (46). The utility of trilinear propagation is that it represents the dose of several individuals in a single anatomical landmark.

\section{III.4 Assessment metrics of interindividual mapping}

In the area of dose mapping, evaluating the accuracy of interindividual mapping is particularly complex because of the nonexistence of ground truth. In the literature, different measures have been proposed to estimate anatomical mapping accuracy and transformation validity $(19,47-51)$. One of the most commonly used assessment methods involves measuring overlap between the reference structure of the anatomical template and the one registered (52). Dice score and Hausdorff distance are among the most commonly used evaluation metrics. However, caution should be exercised when interpreting such measures, which only reflect overall geometric overlap between transformed structures and do not show local mapping errors within the structure. It has been demonstrated that they are not very reliable for locally assessing mapping accuracy, given that a high overlap score does not necessarily imply good point-to-point mapping (53). 
In the setting of population analysis, a mapping error may lead to very different results in statistical analysis. Differences in regions of homogeneous dose are of no consequence for statistical analysis. Conversely, small differences in a region with a steep dose gradient may result in large differences in dose on the reference rectum. Such a situation is described in Figure 3 illustrating two scenarios (1) and (2). Let $R$ be a patient's rectum and $T_{S}(R)$ its image after transformation $T_{S}, s \in\{1,2\}$. The two transformations result in the same difference in alignment $d x$ but in two opposite directions. However, the dose to the anatomical template rectum $R_{A T}$ differs across the two scenarios: in the first scenario, the mapping error leads to a map with higher doses (scenario 1), and in the second, to a map with lower doses.

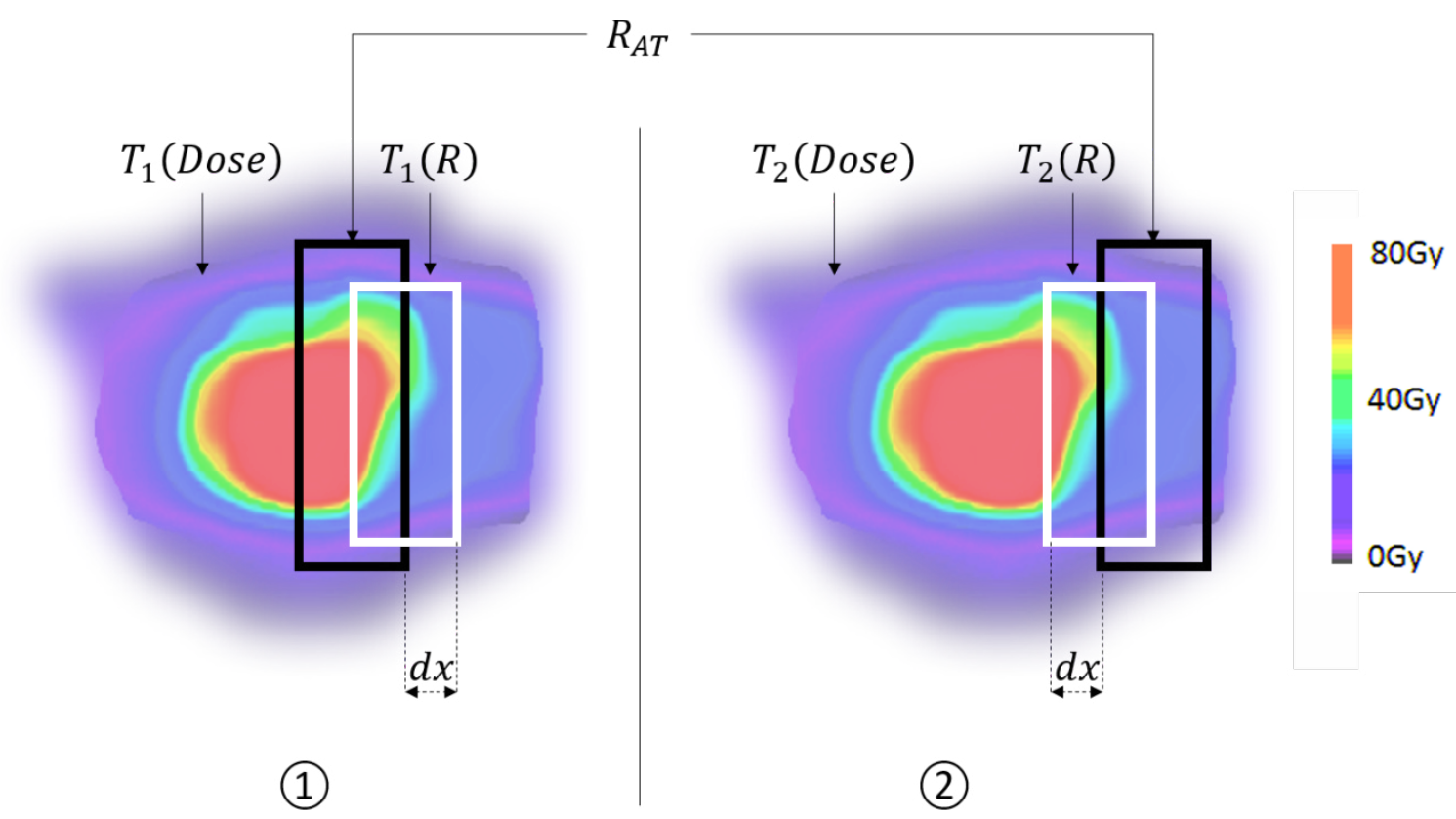

Figure 3: Two mapping processes leading to a different map of the dose (isodose curves $d_{i} G y$ ) to the anatomical template rectum.

In addition to the metrics conventionally used to assess registration, we propose here a metric based on distribution that enables in-depth evaluation. The dice score was used (54) expressed as:

$$
\operatorname{Dice}\left(V_{F}, V_{M}\right)=2 \cdot \frac{\left|V_{F} \cap V_{M}\right|}{\left|V_{F}\right|+\left|V_{M}\right|}
$$

with $V_{F}$ as the anatomical template volume, $V_{M}$ the registered volume and $|\cdot|=\operatorname{card}(\cdot)$, that is, number of voxels of the volume. Another assessment metric is the Hausdorff distance $H D$ (55) which measures the maximum distance between these structures according to: 


$$
H D\left(V_{F}, V_{M}\right)=\max \left\{h\left(V_{F}, V_{M}\right), h\left(V_{M}, V_{F}\right)\right\}
$$

with:

$$
h\left(V_{F}, V_{M}\right)=\max _{f \in V_{F}} \min _{m \in V_{M}}\left\|V_{F}-V_{M}\right\|
$$

The behavior of the inside of the rectum was also analyzed by measuring rectal centerline dispersion $\gamma_{i}$ after registration. In practice, mean centerline dispersion (MCD) is a measure of dispersion between the rectal centerline of the anatomical template $\gamma_{A T}$ and the registered rectal centerline of the individual $T\left(\gamma_{i}\right)$, which are described using B-splines, and then discretized into $N$ equidistant points (here, $N=30$ ). MCD is then expressed as the mean of the Euclidean distances between corresponding points according the equation:

$$
\operatorname{MCD}\left(\gamma_{A T}, T\left(\gamma_{i}\right)\right)=\frac{1}{N} \sum_{k=1}^{N}\left\|\gamma_{A T}^{k}-T\left(\gamma_{i}\right)^{k}\right\|
$$

with $\gamma^{k}$ being the $\mathrm{k}^{\text {th }}$ point of the centerline. By convention, the points were discretized from the lowest slice to the highest slice of the rectum, which were delineated according to the clinical protocol already explained.

Anatomical mapping scores do not, however, allow to fully assess dose mapping accuracy, as illustrated in Figure 3. We propose a dosimetric assessment metric of registration based on dose distribution: dose-organ overlap (DOO). This metric measures the ratio between dose distribution $T$ (Dose) on the intersection and union of $T(R)$ and $R_{A T}$ thus:

$$
D O O\left(T(\text { Dose }), T(R), R_{A T}\right)=\frac{\sum_{T(R) \cap R_{A T}} T(\text { Dose })(v)}{\sum_{T(R) \cup R_{A T}} T(\text { Dose })(v)}
$$

Where $v$ represents a voxel.

DOO is a value between 0 , when structures have no voxels in common, and 1 , when the dose to the structures is identical. In practical terms, the score penalizes anatomical difference by taking into account the dose that would be mapped onto the reference structure.

\section{III.6 Experiments}

The proposed method was assessed with real data and its performance was compared with that of two other methods in which transformation is calculated using the Demons algorithm. 
A total of 30 patients treated with external radiotherapy for localized prostate cancer were considered for this study. For each of them we had at our disposal treatment planning data obtained according to the same clinical protocol: planning CT, organ delineation (prostate, bladder and rectum), and 3D dose distribution. Each CT image comprised slices of $512 \times 512$ pixels $(1 \mathrm{~mm} \times 1 \mathrm{~mm})$ in the axial plane, with an interslice distance of $2 \mathrm{~mm}$. See supplementary material at [URL will be inserted by AIP] for an example of delineations and planning dose distributions (Figure 7 electronic version).

Using a leave-one-out cross-validation procedure, each of the 30 patients was iteratively chosen as the anatomical template in accordance with the process presented in Figure 1. The same procedure was followed in order to map individuals according to the three other methods being used for comparison purposes. Mapping accuracy was evaluated by means of the dice score, Hausdorff distance, MCD, as well as DOO. In addition, some dosimetric parameters (Dose max, Mean Dose and V72) according to the guidelines for target volume definition in radiotherapy for prostate cancer used in the GETUG06 randomized trial (Beckendorf et al., 2011) were compared before and after dose mapping.

The first comparative method involved nonrigid registration based on tissue density of the pelvic region as displayed by CT images. We will abbreviate this method to CT-Demons from now on.

As for the proposed DMLA method, two other methods involved Euclidean distance to the organ boundary and/or to the organ centerline (56) were included. The first method will be abbreviated to DMDM from now on. In the DMDM method, the distance map came in two forms: (i) the external organ, in the form of Euclidean distance to the organ boundary, and (ii) internal organ, in the form of a normalized distance map relating to the anatomical template's structure to be registered. Normalization was required so that the maps of very different individuals, particularly in terms of rectal volume, might be compared. Thus, considering an individual $i$ and the anatomical template $A T$, the descriptor maps $\Phi_{k, l}$ of this method were:

$$
\Phi_{k, l}(v)=\left\{\begin{array}{l}
\tilde{\delta}_{\mid \text {internal }}^{k, l}(v), \text { if } v \in \text { Rectum } \\
\delta^{k}(v) \text { otherwise }
\end{array}\right.
$$


with: $k \in\{i, A T\}, l$ the complement of $k$ in $\{i, A T\}, v$ a voxel of the image, $\delta^{k}$ the Euclidean distance map to the boundary of the individual's rectum $k$, and $\tilde{\delta}_{\mid \text {internal }}^{k, l}=\max \left\{\delta_{\text {|internal }}^{l}\right\} \times$ $\delta_{\text {|internal }}^{k}$.

The second method will be abbreviated as CLDMDM from now on. In the CLDMDM method, the distance map came in two forms: (i) the external organ, in the form of Euclidean distance to the organ boundary, and (ii) internal organ, in the form of Euclidean distance from the organ centerline Thus, considering an individual $i$ and the anatomical template $A T$, the descriptor maps $C D_{k, l}$ of this method were:

$$
\Phi_{k}(v)=\left\{\begin{array}{l}
-\delta_{\text {Centerline }}^{k}(v), \text { if } v \in \text { Rectum } \\
\delta_{\text {Rectum }}^{k}(v) \text { otherwise }
\end{array}\right.
$$

\section{Results \& Discussion}

Figure 4 illustrates the results of registration between a patient and an anatomical template. The results of the evaluation of the CT-Demons, DMDM and DMLA registration methods are detailed in Figure 5.

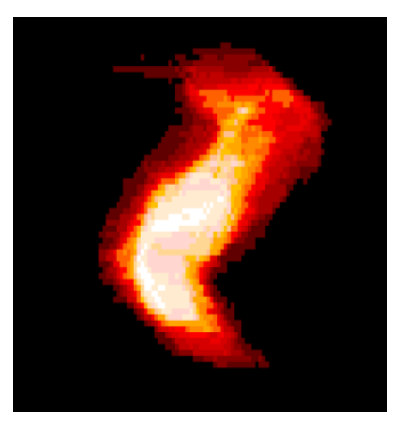

(a) CT-Demons

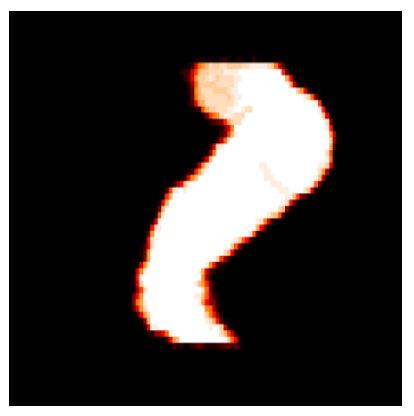

(b) DMDM

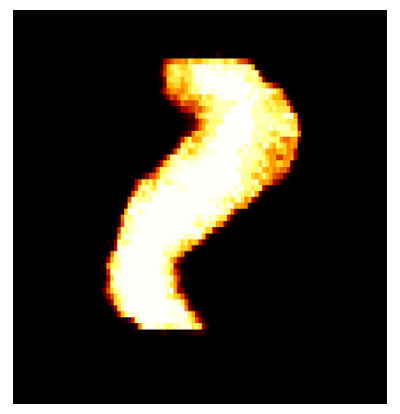

(c) CLDMDM

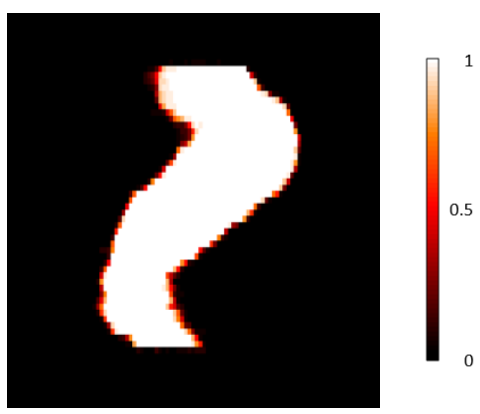

(d) DMLA 

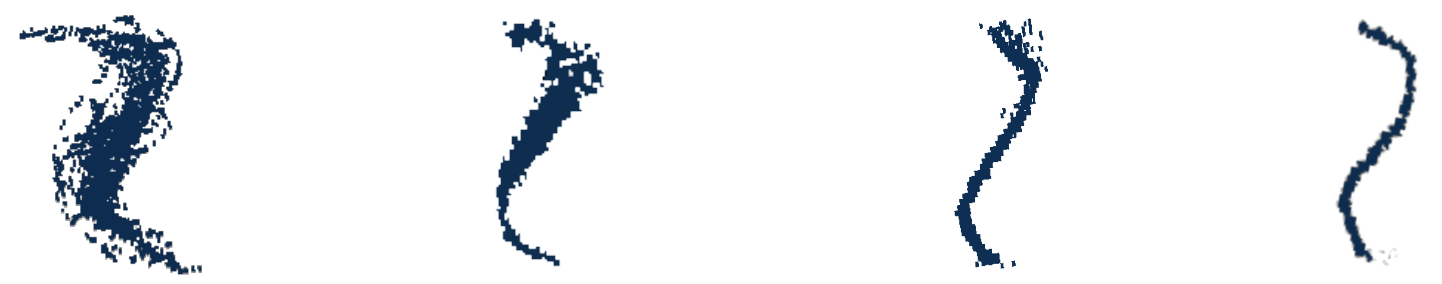

(e) CT-Demons

(f) DMDM

(g) CLDMDM

(h) DMLA

Figure 4: An example of registration results for 29 individuals to the anatomical template: probability maps of the rectum $(a, b, c)$ and representations of centerlines $(d, e, f)$ according to the various compared mapping methods.

The illustration in Figure 4 depicts the probability map of the rectums of 29 individuals using the CTDemons method shows very approximative organ mapping (Figure 4a). In contrast, rectal mapping using the DMDM method (Figure 4b), the CLDMDM method (Figure 4c) or the proposed DMLA method (Figure 4d) appears to be superior This is confirmed by the dice scores and Hausdorff distances calculated in the $29 \times 30$ registrations and illustrated in Figure $5(a-b)$. It can be seen that mapping using the CT-Demons method showed little accuracy since mean dice score was 0.7 and Hausdorff distance was more than $20 \mathrm{~mm}$. The other metrics proposed reinforce this observation. MCD was nearly $18 \mathrm{~mm}$, suggesting an approximative mapping of the inside of the rectum. Similarly, the mean DOO of 0.58 indicates that the dose to the registered organ differed greatly from the dose to the anatomical template organ.

The DMDM, CLDMDM and DMLA methods performed better regardless of the assessment metric taken into consideration. This may be due to the fact that these methods use the same structures (delineation, rectal centerline) that are also used for assessment. Therefore, in order to obtain accurate mapping, it would appear to be wise to use the anatomical information available as much as possible in the registration process, in this case organ delineations.

The results also suggest that the DMLA method yielded significantly more accurate mapping of the rectal structure (Mann-Whitney, $\mathrm{p}<0.05)$ than did the DMDM or CLDMDM methods each metric used. The DMLA and the CLDMDM methods lowered MCD to a mean of less than $6 \mathrm{~mm}$ or $7 \mathrm{~mm}$ respectively as against $12.3 \mathrm{~mm}$ for the DMDM method. This may be due to several factors: (i) First, given how the descriptor maps of the DMDM are constructed, the registration process tends to give precedence to correspondence between the maximum values in the organs. Mapping using the points with the highest values is debatable in pseudocylindrical structures like the rectum. As can be seen in supplementary material at [URL will be inserted by AIP] (Figure 9 electronic), the rectum is a complex pseudo-tubular structure, and the highest value of a distance map of two different rectums 
may not be at the same anatomical location. Matching these points will lead to a biased dose distribution alignment. (ii) Second, rectal centerline is used in order to construct the DMLA and the CLDMDM. This again underlines the importance of assessing mapping accuracy. Whereas the dice score and Hausdorff distance appear to be close (although significantly different) for the DMDM and DMLA methods, a closer evaluation reveals a very different standard of centerline mapping via MCD. On the contrary, whereas MCD appear to be close for the CLDMDM and DMLA methods, the other metrics revealed high differences, suggesting that the countours of the rectum was not as well as mapped than with the DMLA method. This could be explained by the way the descriptors map of the CLDMDM was computed: because the high interindividual variability of the rectum across a population, the distance map from the centerline inside the rectum may present also high variability. Thus, as opposed to the DMDM and CLDMDM methods, the proposed DMLA method enables to compute a normalized structural descriptors map which considers simultaneously both the contours and the centerline of the organ. For instance, with a Demons registration, the relationships between dice score and, respectively, MCD and DOO are shown in Figure 5. No relationship was observed between dice score and MCD. For example, it was possible for a single dice score of around 0.8 to correspond to a relatively low $(<7 \mathrm{~mm})$ or very high $(>30 \mathrm{~mm}) \mathrm{MCD}$. Conversely, there seemed to exist a relationship between DOO and dice score. However, DOO appears to penalize inaccurate registration more. Between dice score and DOO there was a drop of $16 \%$ with CT-Demons, as against $5 \%, 9 \%$ and $4 \%$ with the DMDM, CLDMDM and DMLA methods respectively.

In light of these results, DMLA would appear to be better indicated for in-depth analysis of dose distribution within the rectum than the other methods tested. This method could also be applied in ellipsoidal organs such as the prostate and bladder by using the organ's center of gravity rather than the centerline, as with the rectum.

Table 1 shows the averaged differences in Dose Max, V72 and Mean Dose before and after non-rigid registration on a population of 29 pts registered to a single template. The lower differences appear with the proposed DMLA method. 


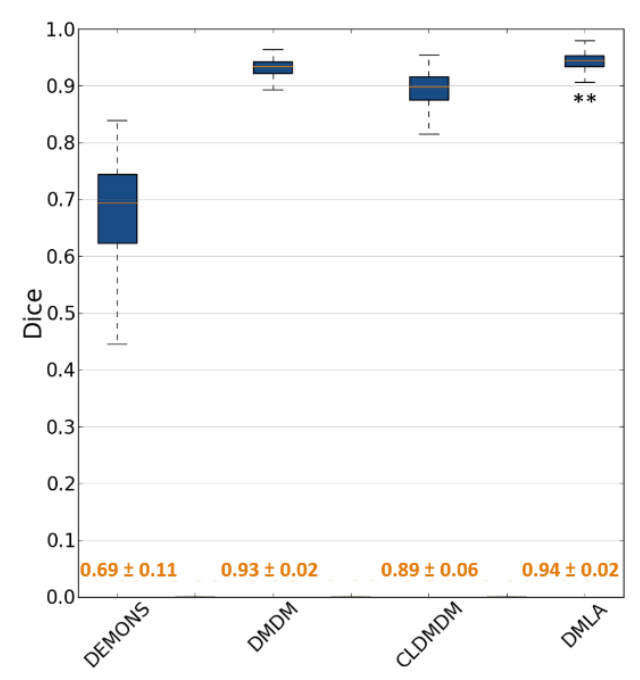

(a) Dice score

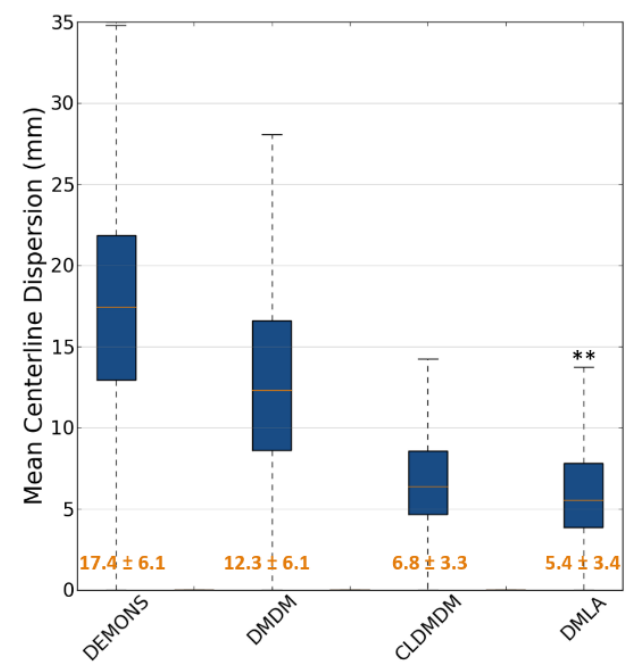

(c) Mean centerline dispersion ( $\mathrm{mm}$ )

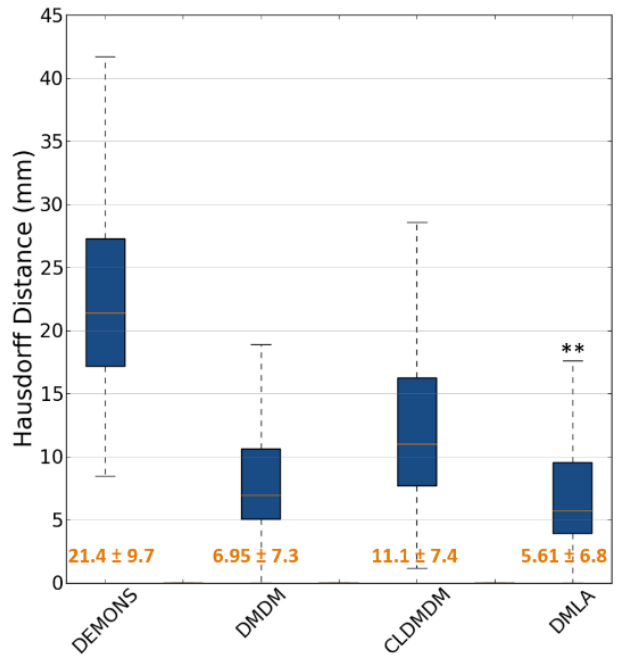

(b) Hausdorff distance ( $\mathrm{mm}$ )

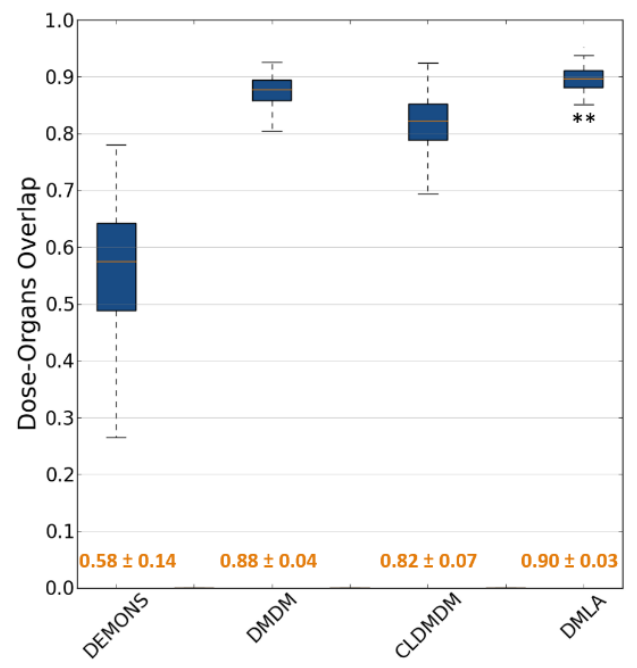

(d) Dose-organ overlap

Figure 5: Summarized results for each metric (mean \pm standard deviation, ${ }^{* *}$ significant difference on MannWhitney test) for CT-Demons, DMDM and DMLA. A) Dice score (biggest close to 1 is the best); b) Hausdorff distance (smallest close to zero is the best); c) MCD (smallest is the best); d) DOO (biggest close to 1 is the best)

\begin{tabular}{|l|r|r|r|r|}
\hline Differences & CT Demons & \multicolumn{1}{c|}{ DMDM } & CLDMDM & \multicolumn{1}{c|}{ DMLA } \\
\hline Dose Max (Gy) & 7.149 & 1.011 & 0.971 & $\mathbf{0 . 8 1 2}$ \\
\hline$V_{72}$ & 3.484 & 1.953 & 1.974 & $\mathbf{1 . 8 8 2}$ \\
\hline Dose Mean (Gy) & 4.023 & 2.819 & 2.802 & $\mathbf{2 . 7 8 3}$ \\
\hline
\end{tabular}

Table 1. Averaged differences in Dose $\max , \mathrm{V}_{72}$ and Dose Mean, computed before and after registration over a population of 29 individuals being treated with the same RT clinical protocol. The proposed DMLA is the method that better preserves the DVH dosimetric parameters. 
The approach that we propose aims at an in-depth analysis of the local dose-effect relationship (1416) as made possible by accurate interindividual mapping using the DMLA registration method presented in this paper. The assessment of this method suggests that rectal mapping using the DMLA method enables an entire population of rectums to be mapped in a sufficiently accurate fashion so that dose distribution may be statistically compared voxel by voxel. We thereby ensured that the results of population analysis would be anatomically meaningful. Under these assumptions, it would be then possible to calculate for example the mean in each voxel by group in patients with and statistical analysis so as to identify the rectal subregions correlating with the occurrence of sideeffects.

As an example of voxel-wize operations, Figure 6 depicts the averaged 3D dose distribution of 29 patients after being mapped to a single template using three different registration methods. It has to be noticed that registration may impact voxel-wise computation as the dose may be differently mapped.

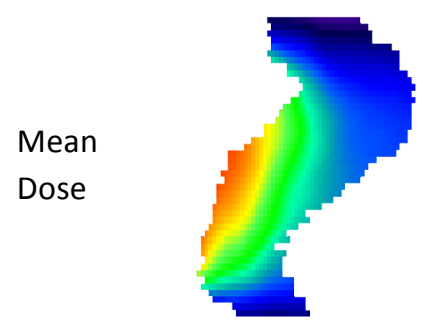

a) Demons

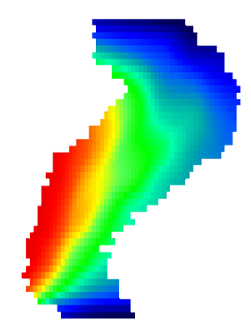

b) DMDM

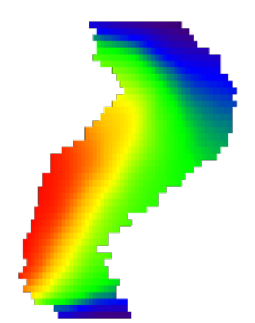

c) CLDMDM

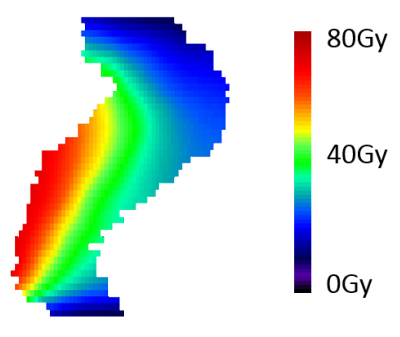

d) proposed DMLA

Figure 6. Sagittal view of mean 3D dose distribution in the rectum from a cohort of 29 individuals after being mapped to a single anatomical template. The voxel-wise mean was computed following three different registration methods: a) Intensity-based Demons, b) DMDM, c) CLDMDM and d) the proposed DMLA.

In this paper, we address the problem of interindividual rectal mapping so as to analyze voxel by voxel the relationship between the occurrence of a toxicity event and planned dose within the rectum. Voxel-wise analysis may be applied to any of the organs at risk in order to study toxicity events. The rectum was chosen here over the bladder for two reasons: First, because dose-effect 
relationships have been established for this organ and rectal toxicity in particular has been studied in the literature (57-61). Second, the rectum deforms less from one session to the next during treatment than the bladder does. The utility of a study based on planned dose would therefore be more debatable in the bladder. Furthermore, the decision to study dose within the rectum rather than at the organ's surface was made because dose/volume constraints during planning apply to the organ in its entirety (7).

The proposed method can also be applied for registration of any tubular structures, relying on the central path and whole organ description. In case of intra-individual rectum registration, for instance, the method can be also be used for computation of cumulative dose in an image guided radiotherapy setup.The laplacian field, provides a way to map doses inside a hollow organ such as the rectum, where the gradient of dose may be important and the DVH although informative, does not provide a description of the 3D dose distribution. The main contribution brought by the Laplacien field is the computation of a normalized structural description which is comparable across individuals, as opposed to classical idstance maps.

Although From a macroscopic point of view, Dmax, Dose moy, V72 may be equivalent, the 3D distribution might not be equivalent thereby potentially leading to quite considerable different results when performing analysis of local dose.The proposed scores allows the computation of the error within the structure, penalizing mismatching in regions with an important gradient of dose

\section{Conclusion}

In our paper, we have proposed a novel interindividual mapping method for the rectum. The main contribution of our research is the creation of a 3D structural model of the rectum based simulteanously on the centerline and the countours and Laplacian field, which provides a normalized descriptor so as to make the most of the rectum's tubular shape. The proposed method makes it possible to map both the rectum's contours and its centerline. Our results indicate that using anatomical/geometrical information during the registration process significantly improves mapping accuracy.

This paper paves the way for voxel-wise population comparisons of dose distribution and may enable local dose-effect relationships to be analyzed in depth. This may make it possible to identify at-risk regions that need to be avoided during radiotherapy treatment planning, thereby improve radiotherapy's reliability. 
See supplementary material at [URL will be inserted by AIP] for an example of planning dose distribution, laplacian construction and distance maps for different individuals.

*Author to whom correspondence should be addressed. Electronic mail:

1. Zietman AL, Bae K, Slater JD, Shipley WU, Efstathiou JA, Coen JJ, et al. Randomized Trial Comparing Conventional-Dose With High-Dose Conformal Radiation Therapy in Early-Stage Adenocarcinoma of the Prostate: Long-Term Results From Proton Radiation Oncology Group/American College of Radiology 95-09. J Clin Oncol. 2010 Mar 1;28(7):1106-11.

2. Zietman AL, DeSilvio ML, Slater JD, Rossi CJ, Miller DW, Adams JA, et al. Comparison of conventional-dose vs high-dose conformal radiation therapy in clinically localized adenocarcinoma of the prostate: a randomized controlled trial. Jama. 2005;294(10):1233-9.

3. Fiorino C, Valdagni R, Rancati T, Sanguineti G. Dose-volume effects for normal tissues in external radiotherapy: Pelvis. Radiother Oncol. 2009 Nov;93(2):153-67.

4. Fonteyne V, Villeirs G, Speleers B, De Neve W, De Wagter C, Lumen N, et al. IntensityModulated Radiotherapy as Primary Therapy for Prostate Cancer: Report on Acute Toxicity After Dose Escalation With Simultaneous Integrated Boost to Intraprostatic Lesion. Int J Radiat Oncol. 2008 Nov 1;72(3):799-807.

5. Kutcher GJ, Burman C. Calculation of complication probability factors for non-uniform normal tissue irradiation: The effective volume method gerald. Int J Radiat Oncol. 1989 juin;16(6):1623-30.

6. Kutcher GJ, Burman C, Brewster L, Goitein M, Mohan R. Histogram reduction method for calculating complication probabilities for three-dimensional treatment planning evaluations. Int J Radiat Oncol Biol Phys. 1991 May 15;21(1):137-46.

7. Beckendorf V, Guerif S, Le Prisé E, Cosset J-M, Bougnoux A, Chauvet B, et al. 70 Gy Versus 80 Gy in Localized Prostate Cancer: 5-Year Results of GETUG 06 Randomized Trial. Int J Radiat Oncol. 2011 juillet;80(4):1056-63.

8. Tucker SL, Zhang M, Dong L, Mohan R, Kuban D, Thames HD. Cluster model analysis of late rectal bleeding after IMRT of prostate cancer: A case-control study. Int J Radiat Oncol Biol Phys. 2006;64(4):1255-64.

9. Working IMRTC. Intensity-modulated radiotherapy: current status and issues of interest. Int J Radiat Oncol Biol Phys. 2001;51(4):880-914.

10. Buettner F, Gulliford SL, Webb S, Partridge M. Modeling late rectal toxicities based on a parameterized representation of the 3D dose distribution. Phys Med Biol. 2011 Apr 7;56(7):2103.

11. Buettner F, Gulliford SL, Webb S, Partridge M. Using dose-surface maps to predict radiationinduced rectal bleeding: a neural network approach. Phys Med Biol. 2009 Sep 7;54(17):5139. 
12. Buettner F, Gulliford SL, Webb S, Sydes MR, Dearnaley DP, Partridge M. Assessing correlations between the spatial distribution of the dose to the rectal wall and late rectal toxicity after prostate radiotherapy: an analysis of data from the MRC RT01 trial (ISRCTN 47772397). Phys Med Biol. 2009 Nov 7;54(21):6535.

13. Lee R, Chan EK, Kosztyla R, Liu M, Moiseenko V. Dose-distance metric that predicts late rectal bleeding in patients receiving radical prostate external-beam radiotherapy. Phys Med Biol. 2012 Dec 21;57(24):8297.

14. Acosta O, Drean G, Ospina JD, Simon A, Haigron P, Lafond C, et al. Voxel-based population analysis for correlating local dose and rectal toxicity in prostate cancer radiotherapy. Phys Med Biol. 2013 Apr 21;58(8):2581.

15. Heemsbergen WD, Al-Mamgani A, Witte MG, van Herk M, Pos FJ, Lebesque JV. Urinary Obstruction in Prostate Cancer Patients From the Dutch Trial (68 Gy vs. 78 Gy): Relationships With Local Dose, Acute Effects, and Baseline Characteristics. Int J Radiat Oncol. 2010 Sep $1 ; 78(1): 19-25$.

16. Witte MG, Heemsbergen WD, Bohoslavsky R, Pos FJ, Al-Mamgani A, Lebesque JV, et al. Relating Dose Outside the Prostate With Freedom From Failure in the Dutch Trial 68 Gy vs. 78 Gy. Int J Radiat Oncol. 2010 mai;77(1):131-8.

17. Acosta O, Simon A, Monge F, Commandeur F, Bassirou C, Cazoulat G, et al. Evaluation of multiatlas-based segmentation of CT scans in prostate cancer radiotherapy. In: 2011 IEEE International Symposium on Biomedical Imaging: From Nano to Macro. 2011. p. 1966-9.

18. Dréan G, Acosta O, Simon A, de Crevoisier R, Haigron P. Evaluation of inter-individual pelvic CTscans registration. IRBM. 2011 Nov;32(5):288-92.

19. Castadot P, Lee JA, Parraga A, Geets X, Macq B, Grégoire V. Comparison of 12 deformable registration strategies in adaptive radiation therapy for the treatment of head and neck tumors. Radiother Oncol. 2008;89(1):1-12.

20. Cazoulat G, Simon A, Dumenil A, Gnep K, de Crevoisier R, Acosta-Tamayo O, et al. SurfaceConstrained Nonrigid Registration for Dose Monitoring in Prostate Cancer Radiotherapy. IEEE Trans Med Imaging. 2014; Early Access Online.

21. Greene WH, Chelikani S, Purushothaman K, Knisely JPS, Chen Z, Papademetris X, et al. Constrained non-rigid registration for use in image-guided adaptive radiotherapy. Med Image Anal. 2009;13(5):809-17.

22. Brock KK. Results of a multi-institution deformable registration accuracy study (MIDRAS). Int J Radiat Oncol Biol Phys. 2010;76(2):583-96.

23. Nithiananthan S, Schafer S, Uneri A, Mirota DJ, Stayman JW, Zbijewski W, et al. Demons deformable registration of $\mathrm{CT}$ and cone-beam $\mathrm{CT}$ using an iterative intensity matching approach. Med Phys. 2011;38:1785.

24. Wang H, Dong L, O'Daniel J, Mohan R, Garden AS, Ang KK, et al. Validation of an accelerated'demons' algorithm for deformable image registration in radiation therapy. Phys Med Biol. 2005;50(12):2887. 
25. Rivest-Hénault $D$, Dowson N, Greer P, Dowling J. Inverse-consistent rigid registration of $C T$ and MR for MR-based planning and adaptive prostate radiation therapy. In: Journal of Physics: Conference Series [Internet]. IOP Publishing; 2014 [cited 2016 Feb 25]. p. 012039. Available from: http://iopscience.iop.org/article/10.1088/1742-6596/489/1/012039/meta

26. Rivest-Hénault D, Greer P, Fripp J, Dowling J. Structure-Guided Nonrigid Registration of CT-MR Pelvis Scans with Large Deformations in MR-Based Image Guided Radiation Therapy. In: Clinical Image-Based Procedures Translational Research in Medical Imaging [Internet]. Springer; 2013 [cited 2016 Feb 25]. p. 65-73. Available from: http://link.springer.com/chapter/10.1007/978-3319-05666-1_9

27. Cazoulat G, Simon A, Acosta O, Ospina JD, Gnep K, Viard R, et al. Dose monitoring in prostate cancer radiotherapy using $\mathrm{CBCT}$ to $\mathrm{CT}$ constrained elastic image registration. In: Prostate Cancer Imaging Image Analysis and Image-Guided Interventions [Internet]. Springer; 2011 [cited 2013 Nov 2]. p. 70-9. Available from: http://link.springer.com/chapter/10.1007/978-3-642-23944$1 \_8$

28. Chen T, Kim S, Goyal S, Jabbour S, Zhou J, Rajagopal G, et al. Object-constrained meshless deformable algorithm for high speed 3D nonrigid registration between CT and CBCT. Med Phys. 2010;37:197.

29. Kim J, Kumar S, Liu C, Zhong H, Pradhan D, Shah M, et al. A novel approach for establishing benchmark $\mathrm{CBCT} / \mathrm{CT}$ deformable image registrations in prostate cancer radiotherapy. Phys Med Biol. 2013;58(22):8077.

30. Ou Y, Sotiras A, Paragios N, Davatzikos C. DRAMMS: Deformable registration via attribute matching and mutual-saliency weighting. Med Image Anal. 2011;15(4):622-39.

31. Weistrand $\mathrm{O}$, Svensson $\mathrm{S}$. The ANACONDA algorithm for deformable image registration in radiotherapy. Med Phys. 2015;42(1):40-53.

32. Jones SE, Buchbinder BR, Aharon I. Three-dimensional mapping of cortical thickness using Laplace's Equation. Hum Brain Mapp. 2000;11(1):12-32.

33. Lee TC, Kashyap RL, Chu CN. Building Skeleton Models via 3-D Medial Surface Axis Thinning Algorithms. CVGIP Graph Models Image Process. 1994 Nov;56(6):462-78.

34. Pudney C. Distance-ordered homotopic thinning: a skeletonization algorithm for 3D digital images. Comput Vis Image Underst. 1998;72(3):404-13.

35. Deschamps T, Cohen LD. Fast extraction of tubular and tree 3D surfaces with front propagation methods. In: Pattern Recognition, 2002 Proceedings 16th International Conference on [Internet]. 2002 [cited 2013 Dec 5]. p. 731-4. Available from: http://ieeexplore.ieee.org/xpls/abs_all.jsp?arnumber=1044862

36. Deschamps T, Cohen LD. Fast extraction of minimal paths in 3D images and applications to virtual endoscopy. Med Image Anal. 2001;5(4):281-99.

37. Bouix S, Siddiqi K, Tannenbaum A. Flux driven automatic centerline extraction. Med Image Anal. 2005;9(3):209-21.

38. Lohmann G, Preul C, Hund-Georgiadis M. Morphology-Based Cortical Thickness Estimation. In: Taylor C, Noble JA, editors. Information Processing in Medical Imaging [Internet]. Springer 
Berlin Heidelberg; 2003 [cited 2013 Oct 2]. p. 89-100. (Lecture Notes in Computer Science). Available from: http://link.springer.com/chapter/10.1007/978-3-540-45087-0_8

39. Danielsson P-E. Euclidean distance mapping. Comput Graph Image Process. 1980 Nov;14(3):227-48.

40. Rocha KR, Yezzi AJ, Prince JL. A hybrid Eulerian-Lagrangian approach for thickness, correspondence, and gridding of annular tissues. Image Process IEEE Trans On. 2007;16(3):63648.

41. Yezzi Jr AJ, Prince JL. An Eulerian PDE approach for computing tissue thickness. Med Imaging IEEE Trans On. 2003;22(10):1332-9.

42. De Visser H, Passenger J, Conlan D, Russ C, Hellier D, Cheng M, et al. Developing a next generation colonoscopy simulator. Int J Image Graph. 2010;10(02):203-17.

43. Passenger J, Acosta O, de Visser H, Bauer S, Russ C, Ourselin S. Texture coordinate generation of colonic surface meshes for surgical simulation. In: 5th IEEE International Symposium on Biomedical Imaging: From Nano to Macro, 2008 ISBI 2008. 2008. p. 640-3.

44. Yezzi A, Prince JL. A PDE approach for thickness, correspondence, and gridding of annular tissues. In: Computer Vision-ECCV 2002 [Internet]. Springer; 2006 [cited 2013 Dec 5]. p. 57589. Available from: http://link.springer.com/chapter/10.1007/3-540-47979-1_39

45. Diep T-M, Bourgeat $P$, Ourselin S. Efficient use of cerebral cortical thickness to correct brain MR segmentation. In: Biomedical Imaging: From Nano to Macro, 2007 ISBI 2007 4th IEEE International Symposium on [Internet]. 2007 [cited 2013 Dec 5]. p. 592-5. Available from: http://ieeexplore.ieee.org/xpls/abs_all.jsp?arnumber $=4193355$

46. Zhong H, Siebers JV. Monte Carlo dose mapping on deforming anatomy. Phys Med Biol. 2009;54(19):5815.

47. Kaus MR, Brock KK, Pekar V, Dawson LA, Nichol AM, Jaffray DA. Assessment of a model-based deformable image registration approach for radiation therapy planning. Int J Radiat Oncol Biol Phys. 2007;68(2):572-80.

48. Klein A, Andersson J, Ardekani BA, Ashburner J, Avants B, Chiang M-C, et al. Evaluation of 14 nonlinear deformation algorithms applied to human brain MRI registration. Neuroimage. 2009;46(3):786-802.

49. Klein S, Staring M, Pluim JP. Evaluation of optimization methods for nonrigid medical image registration using mutual information and B-splines. Image Process IEEE Trans On. 2007;16(12):2879-90.

50. Lu C, Chelikani S, Papademetris X, Staib L, Duncan J. Constrained non-rigid registration using lagrange multipliers for application in prostate radiotherapy. In: Computer Vision and Pattern Recognition Workshops (CVPRW), 2010 IEEE Computer Society Conference on [Internet]. 2010 [cited 2013 Oct 31]. p. 133-8. Available from: http://ieeexplore.ieee.org/xpls/abs_all.jsp?arnumber $=5543137$

51. Zhong H, Peters T, Siebers JV. FEM-based evaluation of deformable image registration for radiation therapy. Phys Med Biol. 2007;52(16):4721. 
52. Crum WR, Camara O, Hill DL. Generalized overlap measures for evaluation and validation in medical image analysis. Med Imaging IEEE Trans On. 2006;25(11):1451-61.

53. Rohlfing T. Image similarity and tissue overlaps as surrogates for image registration accuracy: widely used but unreliable. Med Imaging IEEE Trans On. 2012;31(2):153-63.

54. Zijdenbos AP, Dawant BM, Margolin RA, Palmer AC. Morphometric analysis of white matter lesions in MR images: method and validation. IEEE Trans Med Imaging. 1994;13(4):716-24.

55. Hausdorff F. Dimension und äußeres Maß. Math Ann. 1918 Mar 1;79(1-2):157-79.

56. Drean G, Acosta O, Simon A, de Crevoisier R, Haigron P. Inter-individual organ-driven CT registration for dose mapping in prostate cancer radiotherapy. In: 2012 9th IEEE International Symposium on Biomedical Imaging (ISBI). 2012. p. 370-3.

57. Benk VA, Adams JA, Shipley WU, Urie MM, McManus PL, Efird JT, et al. Late rectal bleeding following combined $x$-ray and proton high dose irradiation for patients with stages T3-T4 prostate carcinoma. Int J Radiat Oncol. 1993 juin;26(3):551-7.

58. Fiorino C, Rancati T, Valdagni R. Predictive models of toxicity in external radiotherapy. Cancer. 2009;115(S13):3135-40.

59. Marzi S, Arcangeli G, Saracino B, Petrongari MG, Bruzzaniti V, laccarino G, et al. Relationships Between Rectal Wall Dose-Volume Constraints and Radiobiologic Indices of Toxicity for Patients With Prostate Cancer. Int J Radiat Oncol. 2007 mai;68(1):41-9.

60. Peeters STH, Hoogeman MS, Heemsbergen WD, Hart AAM, Koper PCM, Lebesque JV. Rectal bleeding, fecal incontinence, and high stool frequency after conformal radiotherapy for prostate cancer: Normal tissue complication probability modeling. Int J Radiat Oncol. 2006 Sep 1;66(1):11-9.

61. Rancati T, Fiorino C, Gagliardi G, Cattaneo GM, Sanguineti G, Borca VC, et al. Fitting late rectal bleeding data using different NTCP models: results from an Italian multi-centric study (AIROPROS0101). Radiother Oncol. 2004 Oct;73(1):21-32. 


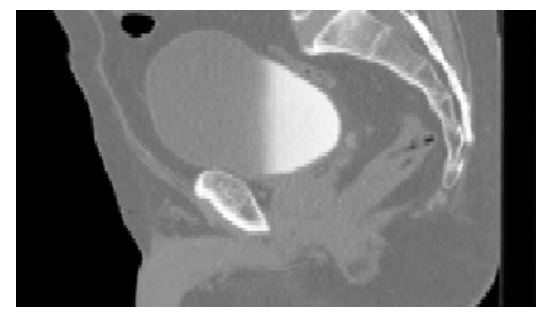

(a) CT Patient 1

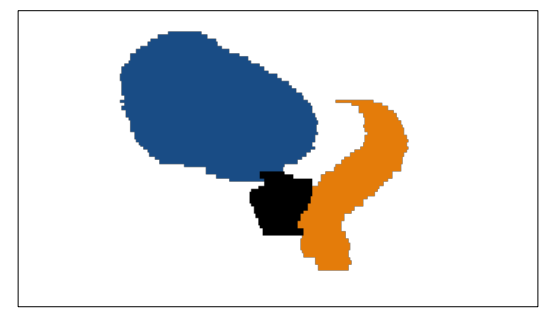

(c) Organs Patient 1

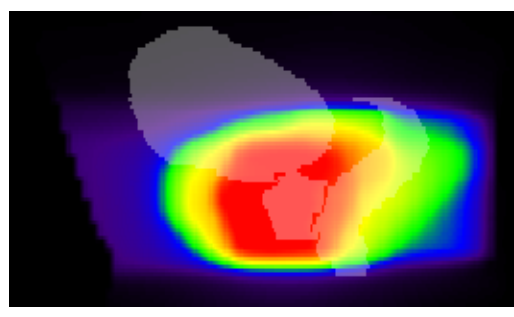

(e) Dose Patient 1

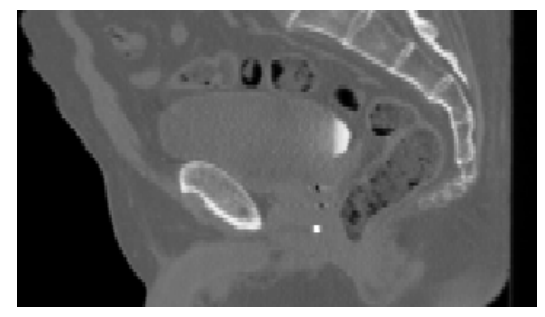

(b) CT Patient 2

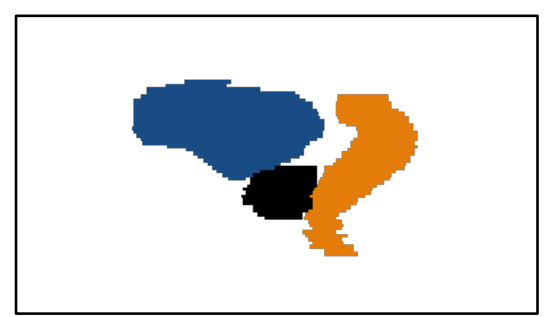

(d) Organs Patient 2

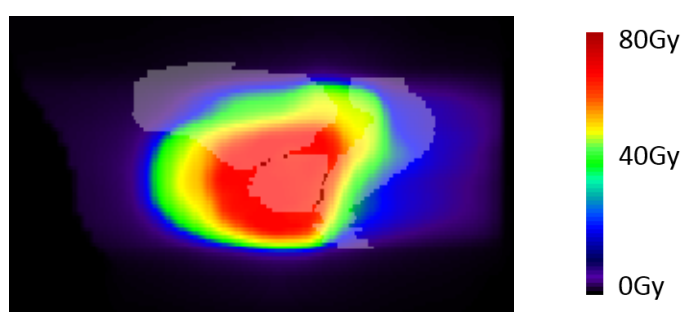

(f) Dose Patient 2

647 Figure 7: Example CTs of two different anatomies (a-b), organ segmentations (prostate [black], bladder 648 [blue], rectum [orange]) and 3D dose distributions of two patients. 


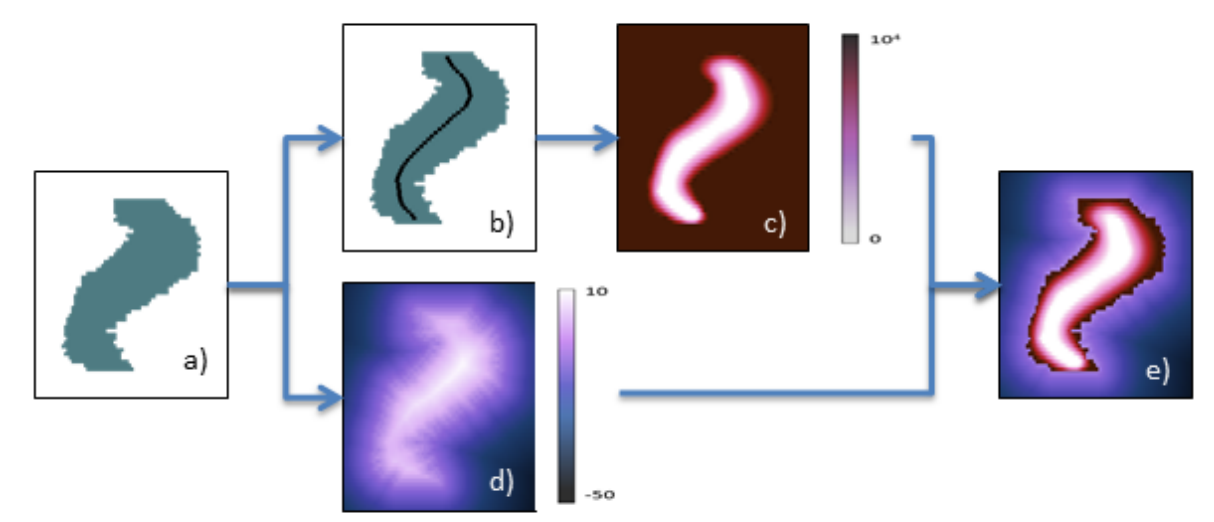

651

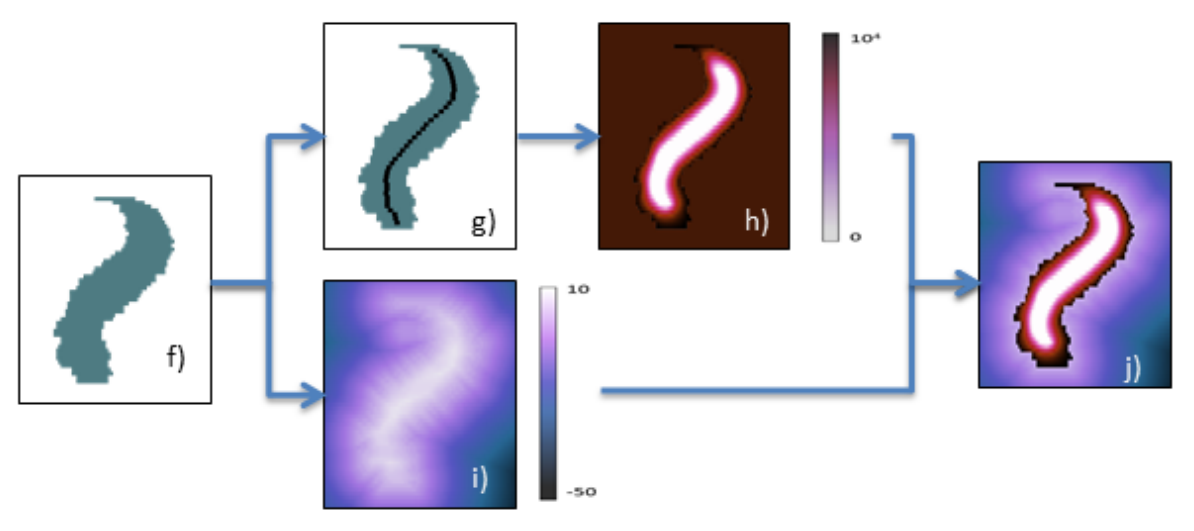

653

654

Figure 8: Example calculations of the 3D structural models obtained for two different rectums (a and f):

655 Centerlines $\gamma$ (b-g); Laplace fields $u$ based on the centerline (c-h); Distance maps $\delta$ (d and i); and 3D 656 structural models $\Phi(\mathrm{e}-\mathrm{j})$. 

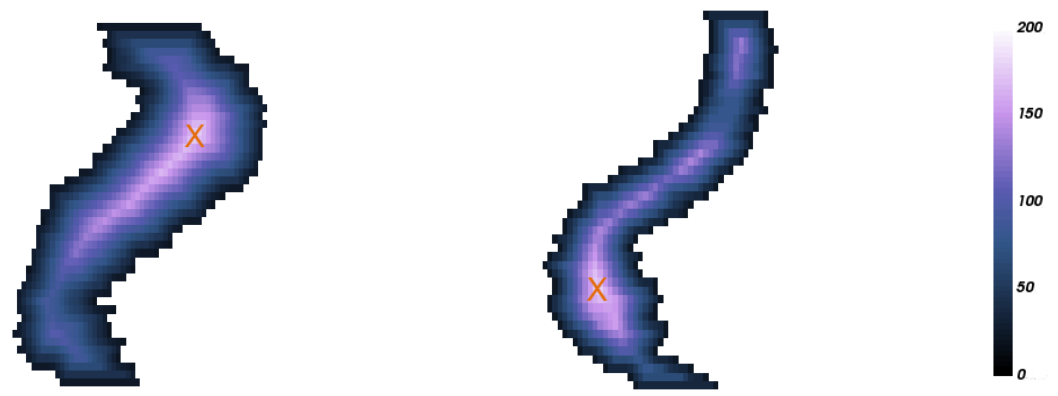

659 Figure 9: Example of normalized distance maps of two rectums (in millimeters). The anatomical locations 660 within the rectum of distance map maximum values (identified with orange Xs) differ.

661 